\title{
ON SELKOW'S BOUND ON THE INDEPENDENCE NUMBER OF GRAPHS
}

\author{
JOCHEN HARANT \\ AND \\ SAMUel Mohr \\ Ilmenau University of Technology \\ Department of Mathematics \\ Ilmenau, Germany \\ e-mail: jochen.harant@tu-ilmenau.de \\ samuel.mohr@tu-ilmenau.de
}

\begin{abstract}
For a graph $G$ with vertex set $V(G)$ and independence number $\alpha(G)$, Selkow [A Probabilistic lower bound on the independence number of graphs, Discrete Math. 132 (1994) 363-365] established the famous lower bound $\sum_{v \in V(G)} \frac{1}{d(v)+1}\left(1+\max \left\{\frac{d(v)}{d(v)+1}-\sum_{u \in N(v)} \frac{1}{d(u)+1}, 0\right\}\right)$ on $\alpha(G)$, where $N(v)$ and $d(v)=|N(v)|$ denote the neighborhood and the degree of a vertex $v \in V(G)$, respectively. However, Selkow's original proof of this result is incorrect. We give a new probabilistic proof of Selkow's bound here.
\end{abstract}

Keywords: graph, independence number.

2010 Mathematics Subject Classification: 05C69.

\section{REFERENCES}

[1] N. Alon and J.H. Spencer, The Probabilistic Method (Wiley, New York, 1992).

[2] Y. Caro, New Results on the Independence Number (Technical Report, Tel-Aviv University, 1979).

[3] S.M. Selkow, A Probabilistic lower bound on the independence number of graphs, Discrete Math. 132 (1994) 363-365. doi:10.1016/0012-365X(93)00102-B

[4] V.K. Wei, A Lower Bound on the Stability Number of a Simple Graph (Technical Memorandum, TM 81 - 11217 - 9, Bell laboratories, 1981).

Received 20 November 2017 Accepted 18 December 2017 